\title{
High-speed 3D imaging of liquid sheet surfaces using the FP-LIF technique
}

\author{
Adrian Roth*1 ${ }^{* 1}$ Marcel Sapík ${ }^{2}$, Jan Jedelský ${ }^{2}$ and Edouard Berrocal ${ }^{1}$ \\ ${ }^{1}$ Division of Combustion Physics, Department of Physics, Lund University, Lund, Sweden \\ ${ }^{2}$ Faculty of Mechanical Engineering, Brno University of Technology, Brno, Czech Republic \\ ${ }^{*}$ Corresponding author email: adrian.roth@forbrf.Ith.se
}

\begin{abstract}
Accurate visualization and understanding of liquid breakups and dynamics of liquid jets, sheets, blobs and ligaments is important for a wide range of spray applications. Here, temporally resolved 3D measurements are necessary to obtain the complete characterization these processes. The currently available 3D imaging techniques are usually based on time-averaged data and do not have the capability of 3D temporally resolving fast liquid breakup phenomena. We have developed a novel technique called Fringe Projection - Laser Induced Fluorescence, FP-LIF, with the possibility of generating snapshot 3D surface reconstructions of irregular liquid structures, such as liquid sheets, ligaments, blobs and other large liquid bodies. The advantage of this technique is that only a single camera is used for generating 3D data. In this article we push the technique one step forward by using a Photron SA-Z high-speed camera to obtain 3D reconstructions of a hollow cone liquid sheet structure at 20000 frames per second. This liquid sheet is generated from a pressure swirl atomizer. Such new type of data, presented here, are particularly relevant for the validation and improvement of computational spray models and for acquiring detailed insight on the conical sheet development and breakup.
\end{abstract}

\section{Keywords}

3D reconstruction, high-speed, pressure swirl atomizer, fluorescence.

\section{Introduction}

Sprays are used for a wide range of applications including painting and coating surfaces, cooling hot environments, inhaling medicament, producing powders in the food and pharmaceutical industries, dispersing chemicals and injecting liquid fuels into combustion engines. Analyzing how the liquid is injected and understanding the formation of droplets is of interest for optimizing and obtaining an efficient spray system for each of these applications. Since spray formation is a complex three-dimensional process, $2 \mathrm{D}$ imaging cannot by itself give the complete picture and 3D measurements are highly desirable. Studies have demonstrated 3D measurements using Computed Tomography (CT) with transmission images from multiple angles by rotating the nozzle. This has been accomplished using either visible light with structured illumination [1] or X-ray absorption [2]. In both cases, image averaging over multiple spray injections was used in order to obtain a statistical representation of the overall spray structure. Another approach is to use Structured Laser Illumination Planar Imaging (SLIPI) [3, 4] to produce series of $2 \mathrm{D}$ slices at different depths in the spray and then stack these results to get 3D data, as shown in $[5,6]$. These examples of 3D reconstructions from image stacking were also relying on averaged data.

The resulting averaged 3D reconstructions depict the general spray structure, but not the stochastic nature of each spray injection neither the liquid dynamics acting on liquid surfaces. Such information requires obtaining high-speed 3D imaging of single events. This remains a challenging task with only a few examples of achievements in the literature. The first example is the $3 D$ spray measurement over time where CT is performed at $10 \mathrm{kHz}$ frame rate by means of three high-flux rotating anode X-ray tube sources together with three intensified high-speed 


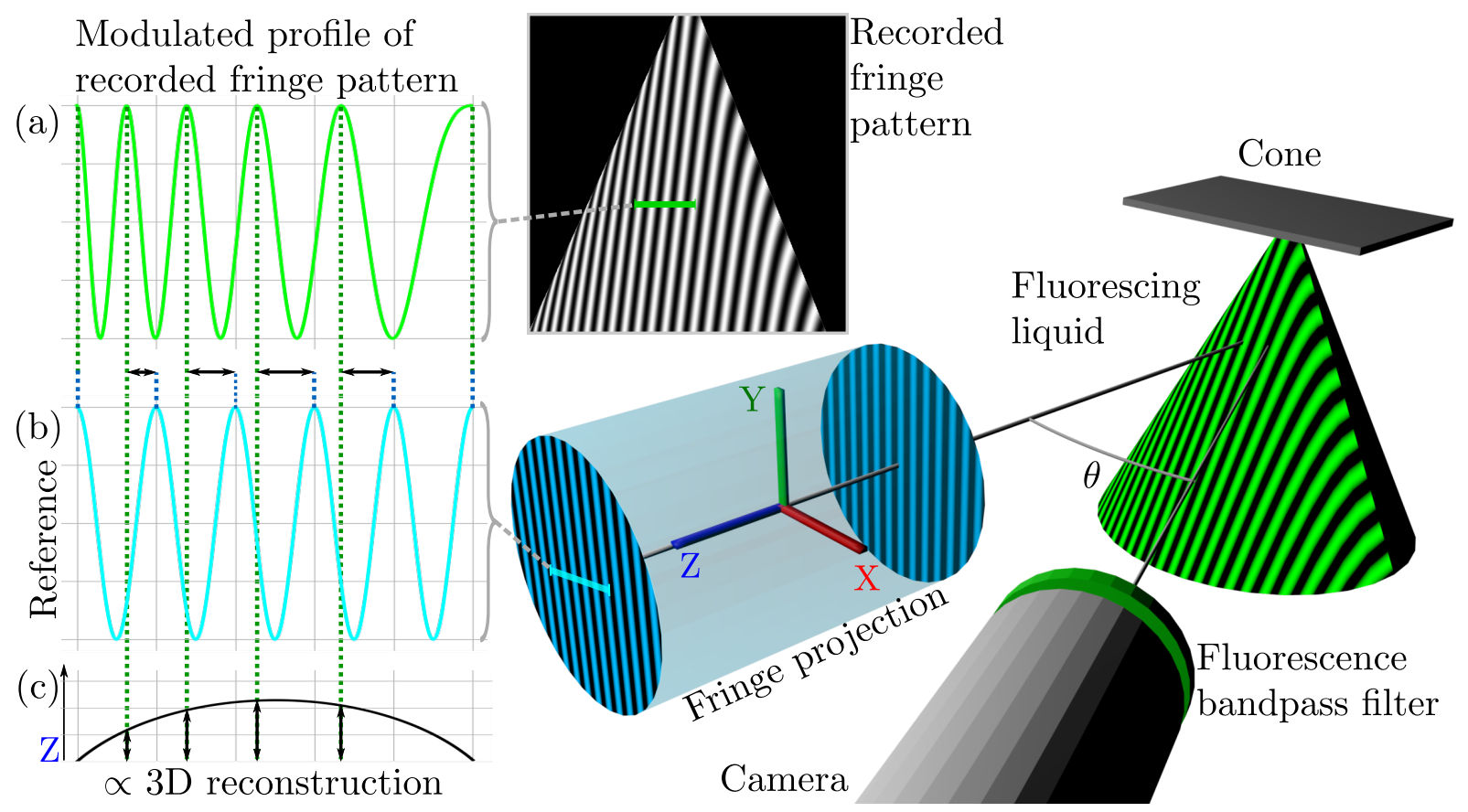

Figure 1. Illustration of the FP-LIF technique. Here the fringe projection illuminates a 3D structure, in this case an example simulated cone, and the fluorescing fringes are imaged from an angle $\theta$ through an optical filter that rejects most of the elastically scattered light. It is visible how the fringes curve on the surface of the liquid and this curvature includes information of the surface topography. This fringe curvature is also illustrated as the change of frequency of the plotted curve on the top left which is the intensity of the imaged fringe projection along the $x$-axis of the image. When the changing frequency of the top curve is compared to the middle curve, a value proportional to the third coordinate is found. With a calibration the absolute 3D coordinates can then be estimated.

cameras [7]. The second example use digital off-axis holography at $20 \mathrm{kHz}$ with a pulsed laser system to investigate the 3D location and sizes of spray drops [8]. The third example use plenoptic imaging as shown in $[9,10]$. In this case an array of micro-lenses is placed at the image plane in front of the sensor. With this optical arrangement the sensor captures image information from a range of observation angles which can be used to extract 3D information. Since the angular information will be at the cost of spatial information, high pixel number is preferred which is troublesome for high-speed imaging. Though a module for high-speed plenoptic $3 \mathrm{D}$ imaging has been recently developed [11].

High-speed 3D imaging of liquid breakups in sprays, showing detailed temporal surface deformation with a fairly good spatial resolution and by means of a single camera remains a challenge. With this goal in mind, we have recently developed a technique called Fringe Projection - Laser Induced Fluorescence (FP-LIF) [12]. FP-LIF is based on the Fringe Projection concept where a structured laser beam is used to illuminate a liquid structure. The injected liquid contains a high concentration of fluorescent dye (Fluorescein in this case) enabling generation of a Laser Induced Fluorescence (LIF) signal close to the liquid surface. From the deformation of the LIF fringes, the topography of the illuminated structure can be extracted. For an illustration of the technique see Figure 1.

The FP-LIF technique is used here with a continuous wave laser illumination and a single Photron SA-Z high-speed camera; providing 3D temporally resolved results at $20 \mathrm{kHz}$ frame rate. To illustrate what information can be found from this data, multiple spray-angles were extracted around the liquid sheet for each 3D reconstruction. Such data generated in this article is valuable for future validation of computational models simulating liquid breakup and for acquiring detailed insight on the phenomena of conical sheet development and breakup. 


\section{Method}

\section{D reconstruction with FP-LIF and Experimental setup}

The FP-LIF technique is a combination of the Digital Fringe Projection (DFP) technique [13] and Laser Induced Fluorescence as the name suggests. DFP is widely used in image analysis to produce 3D reconstructions of object surface geometries. Here, the reconstruction is produced from a single image in contrast to Computed Tomography where multiple views are used. Just as DFP, FP-LIF is based on projecting a periodic fringe pattern of given frequency and detecting the light that is back-scattered from the surface of the object. The fringe projection simply consists of straight lines that repeat at a certain period length $T$, see Figure 1 . To include information of the third dimensions in a single 2D image, the back-scattered signal is imaged with an angle $\theta$ as illustrated in Figure 1 . Now, the recorded image intensity $I$ can in a simplified manner be modeled by the following equation,

$$
I(x, y)=A(x, y)+B(x, y) \cos (\varphi(x, y))+\epsilon(x, y)
$$

Here, $\mathrm{A}$ is the background light intensity in the image, $\mathrm{B}$ is the amplitude of the fringes, epsilon is noise and finally $\phi$ is the phase of the fringes. In this imaged fringe pattern, all interesting information of the third dimension is found in the phase $\phi$ and to extract it a phase demodulation of the image performed, see [12] for more information on this. With the estimated phase, the third dimension, depth $d(x, y)$, can be estimated for the surface with the following equation,

$$
d(x, y)=\frac{T}{2 \pi s \sin (\theta)}\left(\varphi(x, y)-\frac{2 \pi}{T}\left(x-x_{0}\right)\right) .
$$

Here, some parameters are required to be previously calibrated. These include the parameter $T$, which correspond to the width of the fringe projection fringes in pixels. It can be estimated from a calibration image when the fringes are projected onto a flat surface. The scale $s$ of how many pixels there are per meter and finally the principal image point $\left[x_{0}, y_{0}\right]$ of the camera. This is the general approach for the 3D reconstruction using FP-LIF, for more in depth information see [12].

The FP-LIF requires the use of fluorescing liquid. In this experiment Fluorescein was used as fluorescent dye due to its high quantum yield. Here, the excitation wavelength was $450 \mathrm{~nm}$ using a continuous wave collimated diode laser delivering $3 \mathrm{~W}$ power. The beam was extended by a series of spherical lenses reaching approximately $60 \mathrm{~mm}$ in diameter when illuminating the spray, see Figure 2(a). To create the fringe pattern into the beam, a Ronchi grating was inserted along the optical path. In the experiment, a high Fluorescein concentration was used to get a fluorescence signal near the liquid surface. The concentration corresponded to a 1:100 ratio that resulted in an aqueous solution with some liquid properties that notably diverge from pure water. The resulting solution had a surface tension of $57 \mathrm{mN} / \mathrm{m}$, the kinematic viscosity was $0.916 \mathrm{~mm}^{2} / \mathrm{s}$ and the density of the mixture was around $997 \mathrm{~kg} / \mathrm{m}^{3}$. Images were recorded with a Photron SA-Z high-speed camera at 20,000 frames per second (fps) and with a sensor resolution of $1024 \times 1024$ pixels. In order to keep the liquid sheet structure better in focus, the camera objective was mounted with a Scheimpflug imaging configuration.

The liquid was injected at ambient temperature and pressure condition (around $20{ }^{\circ} \mathrm{C}$ and atmospheric pressure) using a pressure swirl atomizer. The atomizer is a $10 \times$ scaled up version of an atomizer developed for an aircraft engine produced in transparent plexiglass [14]. The atomizer scaling and transparency allows detailed optical measurement of the internal flow as well as the liquid sheet formation. This transparent atomizer, see Figure 2(b), contains a swirl chamber with tangential inlet ports and a spill-line at the chamber's top. For a detailed description together with its dimensions, see [15]. The atomizer was operated at a gauge liquid pressure of around 0.2 bar with the spill line closed. 
(a)

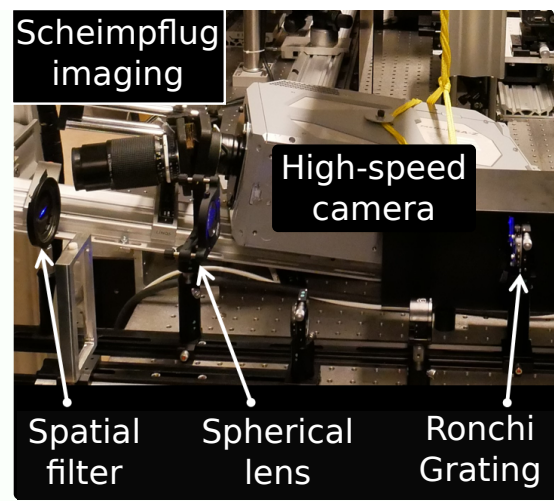

(b)

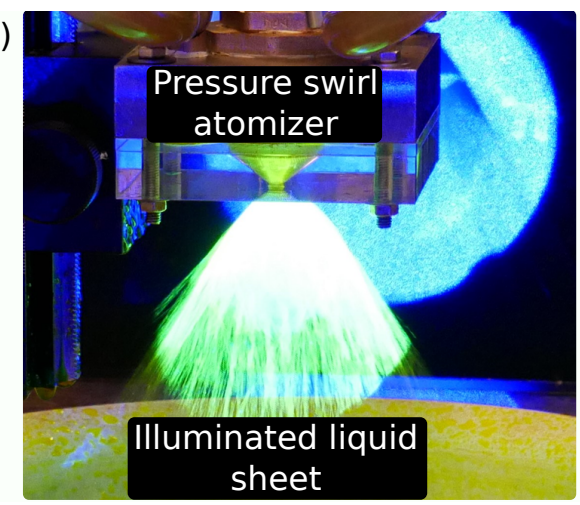

Figure 2. Details of the experiment. (a) Arrangement of the Photron high-speed camera together with respective optical components used to create the laser fringe projection. (b) The illuminated sheet created with the pressure swirl atomizer where green fluorescence from the excited Fluorescein dye is visible from the sheet and the $450 \mathrm{~nm}$ blue laser light can be seen from the light reflected in the background. Note here that the projected fringes are not visible on the liquid as a result of overexposure.

\section{Results and Discussion}

To analyze the hollow cone liquid sheet, 20 consecutive frames of 3D reconstructions were visualized, Figure 3(d). The reconstructed surfaces were virtually imaged using the software Blender. In these reconstructions, phenomena can be observed such as sheet breakup and propagation of surface waves with a time resolution of $0.05 \mathrm{~ms}$ between each reconstruction. These waves will be revisited in the later discussion. In addition, panels (a), (b) and (c) in Figure 3 , show an example FP-LIF image and its corresponding 3D reconstruction.

Prior to each 3D surface reconstruction, a foreground segmentation is required. This can be seen as the brighter area in Figure 3(b). Without this segmentation large errors would be propagated from the dark areas to the real structure during the phase demodulation process. In this work, a manual segmentation was performed for each image which is a time-consuming process, especially with the thousands of images produced by a single high-speed camera recording. It should be noted that we only segmented and 3D reconstructed 100 of these in this work. A more time efficient solution would be an automated segmentation algorithm. Previous work have developed such algorithms [16]. However, the algorithms attempted for the recorded high-speed data in this work was not successful and the failures seem to be connected to the large variation of signal to noise ratio in each of the recorded images together with some bright spots in the images that creates errors in the segmentation. To make the application of the FP-LIF technique useful on data with large number of frames, a robust segmentation algorithm is required. 

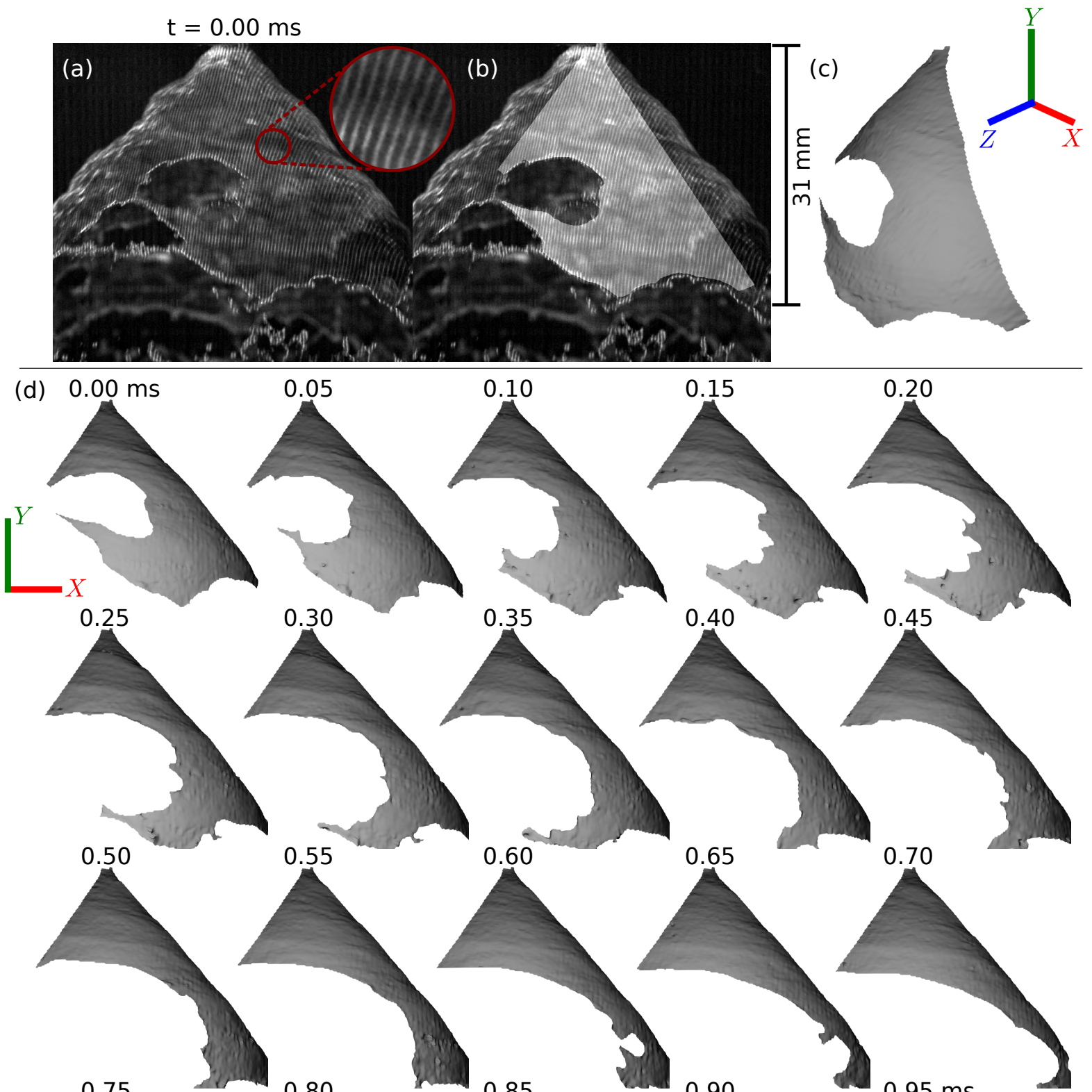

0.55
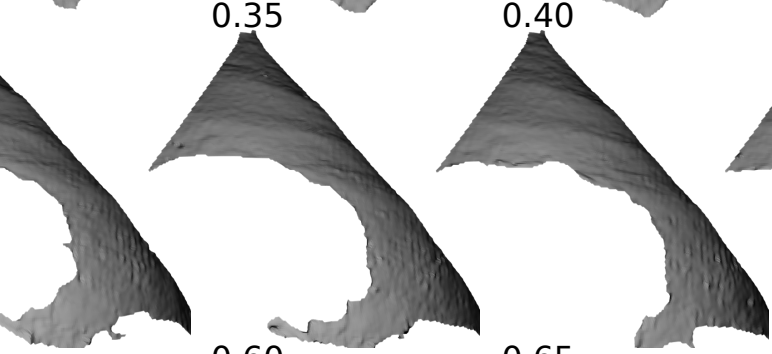

0.45

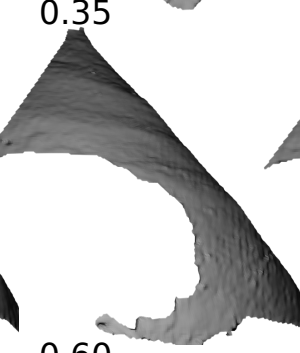

0.05

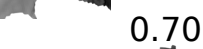

0.70

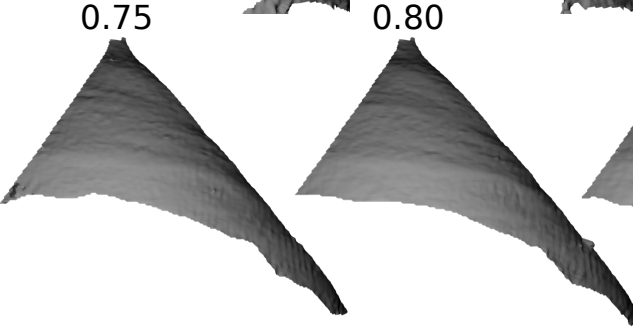

0.85
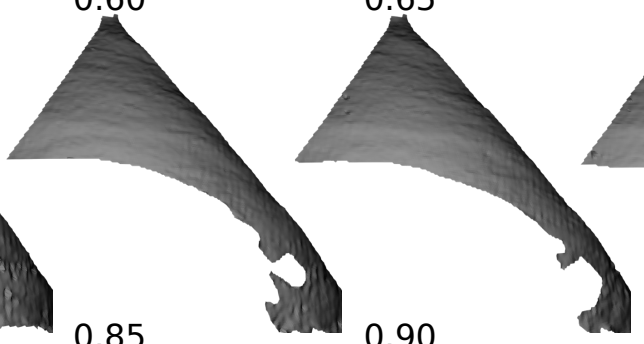

$0.95 \mathrm{~ms}$

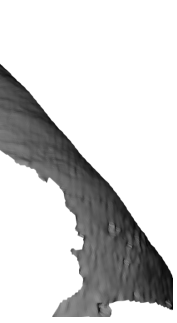



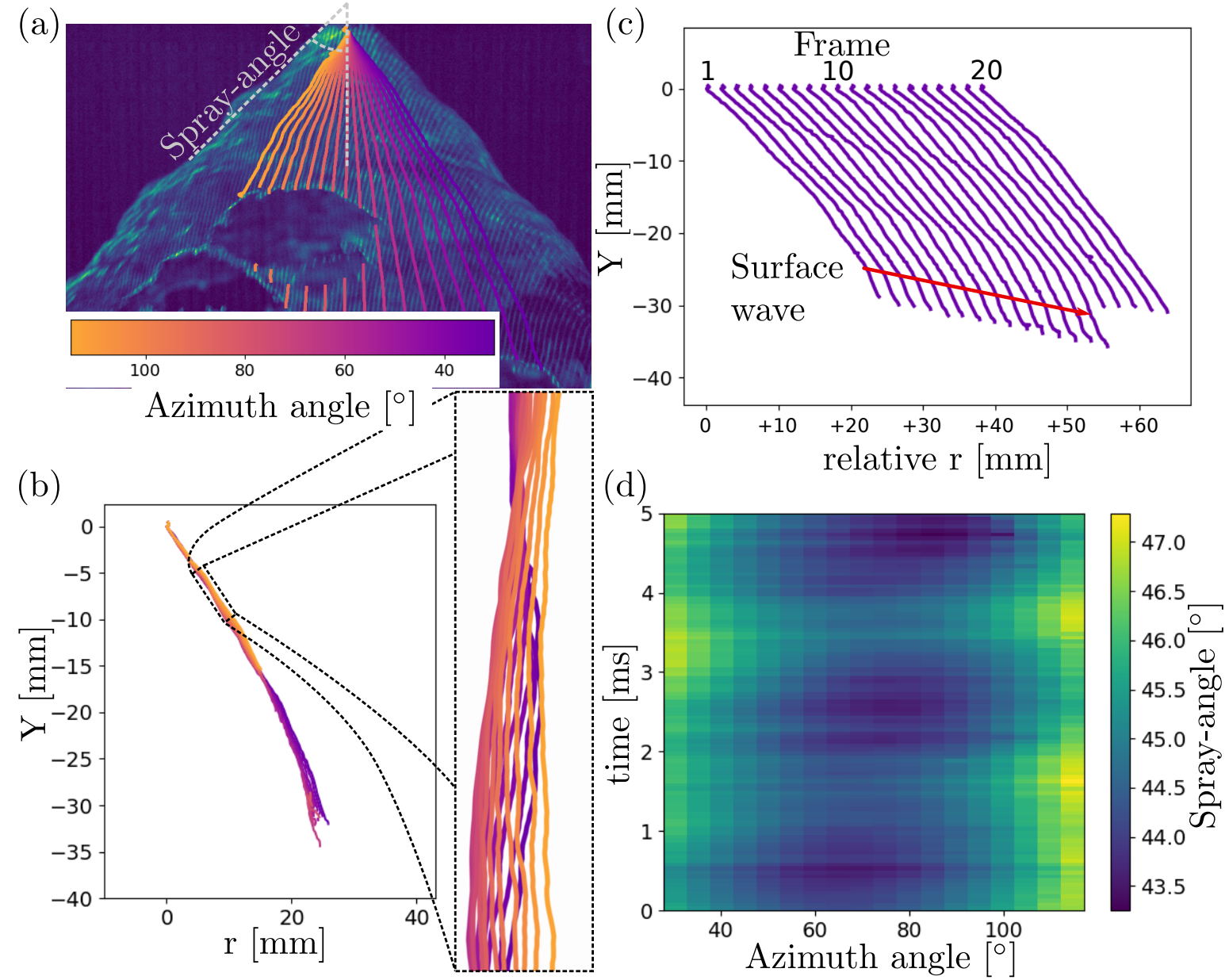

Figure 4. Analysis of high-speed 3D reconstructions. (a) Illustration of how we define both the azimuth angle and spray-angles in an FP-LIF image. (b) Plotted contours of the reconstructed surface at different azimuth angles and one time point where the radius $r=\sqrt{X^{2}+Z^{2}}$. Here, the zoomed in square shows how the surface deviates from a perfect cone. (c) Plotted contours along one azimuth angle over time. Note that the radius here is relative since

each frame has its contour moved $2 \mathrm{~mm}$ to the right for visualization purposes. Here is also a surface wave propagation over time is indicated. (d) The map of estimated spray-angles for 18 different azimuth angles and 100 consecutive frames (a duration of $5 \mathrm{~ms}$ ).

With the 3D reconstructions resolved in time, shown in Figure 3, there are new possibilities for the analysis of liquid structures in general and specifically this hollow cone liquid sheet. In this work some features were investigated with results shown in Figure 4. Panel (a) in this figure shows an image of the liquid sheet that is used for the 3D reconstruction. The colored lines in this image represent azimuth angles in the $X Z$ plane of the 3D coordinate system where the zero angle is in the positive $\mathrm{X}$-direction and 90 degrees is along the positive Z-direction. Panel (b) then shows how the surface contour radius change both along the Y-direction of the spray and for different azimuth angles. Here, the darkest line corresponds to the lowest azimuth angle, and the angle successively gets larger with brighter color. For the zoomed in area one can clearly see the continuous variation of surface contour depending on the azimuth angle. The individual lines indicate that the cone angle noticeably varies around the cones azimuth angle which affects the final droplet trajectory and the change within each line reflects the already mentioned surface waves. The waves' amplitude and velocity can be estimated and used for study of the breakup process, including comparison with theoretical models and CFD results. Here, it should be noted that the larger azimuth angles generally have a lower Signal to Noise Ratio (SNR) which give less precision in the 3D reconstruction. The reason for the lower SNR is connected to a higher frequency of the fringes in the recorded FP-LIF images for these azimuth angles. Panel (c) is similar to (b) but instead of showing different azimuth angles, the 
same azimuth angle is shown over $1 \mathrm{~ms}$ of time. Here, surface waves can be seen and the one indicated in the plot propagates with a speed of around $8 \mathrm{~m} / \mathrm{s}$ along the $Y$ direction. This wave can possibly be connected to the sheet rupture that is occurring after the 15th frame in the figure and it is followed by the sheet breakup. The reconstruction (see the full 100 image sequence https://3d.spray-imaging.com/cone4d/) allows to determine the rupture borders and velocity of its propagation. With higher number of processed images a statistical analysis of the breakup process can be performed.

In Figure 4(d) we have examined the spray-angles of this atomizer in both 3D and over time. This angle is an essential spray characteristic as it defines the dispersion of the liquid in spray applications. Its temporal variations indicate the internal flow stability, and its circumferential symmetry shows the manufacturing precision and possible artifacts of the flow from individual inlet swirling ports. With the 3D reconstructions it is possible to simultaneously analyze this angle along different azimuth angles. The spray-angle for each time point and azimuth angle was calculated by taking the surface contour radius along the $Y$-dimension for values of $Y$ between -15 and $-3 \mathrm{~mm}$. Then, a line was fitted to these values and the angle of the line related to the negative $\mathrm{Y}$-axis was defined as the spray-angle. In Figure 4(d), it can be seen how the angle slightly diverges from axially symmetrical shape where, generally, the azimuth angles 30 and 110 degrees feature a few degrees larger spray-angle compared to the azimuth angle 70 degrees. This elliptical characteristic can be connected to an asymmetry of the nozzle or some kind of bias in the 3D reconstruction algorithm that needs to be further investigated. Otherwise, the spray-angle generally spans between 43 and 48 degrees and, here, patterns with almost "horizontal" fashion are seen. These patterns represent unison fluctuations of the spray-angles over all azimuth angles with a time period of around $2 \mathrm{~ms}$. This fluctuation is probably connected to the surface waves previously mentioned and it indicates a change in the axial to radial velocity ratio that appears during liquid discharge. This is probably caused by instabilities in the liquid delivery (pump pulsations) or by the irregular shape of the air core in the swirl chamber.

\section{Conclusions}

The understanding of liquid structures found in spray systems is important for a wide range of applications and since it is a 3D phenomenon it is of interest to perform detailed 3D measurement on such structures. This measured 3D data will aid in the development and validation of liquid breakup models. In this work, a technique for snapshot 3D reconstruction of liquid structures, called FP-LIF, has been applied in a high-speed measurement where data was recorded with $20000 \mathrm{fps}$. The measurement was performed on a hollow cone liquid sheet structure created by a pressure swirl atomizer where the sheet's surface was reconstructed frame by frame. The hollow cone was then analyzed by looking at surface waves and spray-angles in 3D. Here, the 3D reconstruction enables simultaneous analysis of multiple spray-angles around the cone. The angle was found to vary between 43 and 48 degrees, showing an asymmetrical $3 \mathrm{D}$ reconstruction of the sheet. In addition, an unison pulsating fluctuation of the sheet surface was found with an approximate period of $2 \mathrm{~ms}$ and this fluctuation is probably connected to the surface waves found on the reconstructed surfaces. In the future, there are more possibilities for analysis of sprays from 3D reconstruction with the FP-LIF technique. Here, liquid structures such as jets, sheets, blobs and ligaments can be reconstructed and analyzed to give a better understanding of their formation and evolution.

\section{Acknowledgements}

The authors would like to thank for the financial support from the European Research Council (ERC - 638546), the Swedish Research Council (Vetenskapsrådet 2016-03894) and the program Inter Excellence by the Ministry of Education, Youth and Sports of the Czech Republic (project LTAIN19044). 


\section{Nomenclature}

$\theta \quad$ Angle between illumination and camera direction [degrees]

$X, Y, Z$ World coordinates [m]

\section{References}

[1] E. Kristensson, E. Berrocal, and M. Aldén. Quantitative 3D imaging of scattering media using structured illumination and computed tomography. Opt. Express, 20(13):1443714450, 2012.

[2] L. Marchitto, L. Alocca, D. Hampai, S. Alfuso, S. B. Dabagov, A. Liedl, and C. Polese. 3D structure of liquid sprays: X-ray $\mu$-radiography and tomography by polycapillary based technique. Nuclear Instruments and Methods in Physics Research B, 355:285-288, 2015.

[3] E. Berrocal, E. Kristensson, M. Richter, M. Linne, and M. Aldén. Application of structured illumination for multiple scattering suppression in planar laser imaging of dense sprays. Opt. Express, 16(22):17870-17881, 2008.

[4] E. Kristensson, E. Berrocal, M. Richter, S.-G. Pettersson, and M. Aldén. High-speed structured planar laser illumination for contrast improvement of two-phase flow images. Opt. Lett., 33(23):2752-2754, 2008.

[5] R. Wellander, E. Berrocal, E. Krisensson, M. Richter, and M. Aldén. Three-dimensional measurement of the local extinction coefficient in a dense spray. Meas. Sci. Technol., 22:125303-125318, 2011.

[6] Y. N. Mishra, M. Koegl, K. Baderschneider, B. Hofbeck, E. Berrocal, C. Conrad, S. Will, and L. Zigan. 3D mapping of droplet sauter mean diameter in sprays. Appl. Opt., 58(14):37753783, 2019.

[7] B. R. Halls, N. Rahman, M. N. Slipchenko, J. W. James, A. McMaster, M. D. A. Ligthfoot, J. R. Gord, and T. R. Meyer. 4D spatiotemporal evolution of liquid spray using kilohertzrate x-ray computed tomography. Opt. Lett., 44(20):5013-5016, 2019.

[8] Yingchun Wu, Lei Wang, Wenhui Lin, Ge Song, Yong He, Xuecheng Wu, Zhihua Wang, and Kefa Cen. Picosecond pulsed digital off-axis holography for near-nozzle droplet size and 3D distribution measurement of a swirl kerosene spray. Fuel, 283:119124, January 2021.

[9] P. Lillo, M. Greene, and V. Sick. Plenoptic single-shot 3d imaging of in-cylinder fuel spray geometry. Zeitschrift für Physikalische Chemie, 229, 2015.

[10] E. M. Hall, D. R. Guildenbecher, and B. S. Thurow. Uncertainty characterization of particle location from refocused plenoptic images. Opt. Express, 25(18):21801-21814, 2017.

[11] Z. P. Tan, K. J., C. C., and B. S. Thurow. Development of a modular, high-speed plenopticcamera for 3d flow-measurement. Opt. Express, 27(9):13400-13415, 2019.

[12] A. Roth, E. Kristensson, and E. Berrocal. Snapshot 3d reconstruction of liquid surfaces. Opt. Express, 28(12):17906-17922, Jun 2020.

[13] S. Zhang. High-speed $3 D$ imaging with digital fringe projection techniques. Optical sciences and applications of light. CRC Press, 2016.

[14] J. Jedelský, M. Malý, M. Jícha, J. Sláma, and G. Wigley. Importance of geometrical factors on spray characteristics of spill-return atomizers. Journal of Propulsion and Power, pages 1-11, 2020.

[15] M. Sapík, E. Berrocal, and J. Jedelský. Spray visualization using liquid fluorescence highspeed imaging. Experimental Fluid Mechanics 2019, 2019.

[16] D. C. Ghiglia and M. D. Pritt. Two-Dimensional Phase Unwrapping: Theory, Algorithms, and Software. John Wiley \& Sons, Inc., 1998. 